\title{
AVALIAÇÃO DA EFICÁCIA DE ÓLEOS ESSENCIAIS NO TRATAMENTO DE SEMENTES DE SOJA
}

\author{
Maicon Vinícius Daronco ${ }^{1 *}$, Aline Schneider ${ }^{1}$, Luiz Volney Mattos Viau ${ }^{1}$ e Christiane de Fátima Colet ${ }^{1}$. \\ 'Universidade Regional do Noroeste do Estado do Rio Grande do Sul - UNIJUÍ; \\ *Autor para correspondência: maicon.daronco@hotmail.com
}

RESUMO: Objetivou-se avaliar o efeito dos óleos essenciais (OE) de Cymbopogon flexuosus Stapf, Eucalyptus globulus Labill. e Baccharis trimera (Less) DC. sobre a microflora fitopatogênica e fisiologia de sementes de soja, com aplicação de OE nas concentrações de 10, 20 e 30\%. As análises de germinação e vigor foram realizadas no Laboratório de Análise de sementes da Universidade Regional do Noroeste do Estado do Rio Grande do Sul - UNIJUI e os tratamentos implantados a campo na área experimental do Instituto Regional de Desenvolvimento Rural - IRDeR. Foram avaliadas as variáveis: número inicial e final de plantas sadias, plantas doentes, rendimento de grãos, massa mil de grãos, número de legumes e grãos por planta, peso de grãos por planta; rendimento biológico aparente; índice de colheita. Quanto a análise in vitro das sementes foi verificado: contagem de plântulas germinadas normais; contagem de plântulas germinadas anormais e sementes duras. Foi utilizado análise de variância, com teste de Tukey a $5 \%$ de probabilidade. $O$ tratamento com OE de E. globulus $20 \%$ apresentou o maior rendimento de grãos, com aumento de 10\% em relação ao controle. Quanto à massa de mil grãos, a média entre todos os testes foi de $140 \mathrm{~g}$, não apresentando diferença estatística. Quanto ao número final de plantas de $B$. trimera $30 \%$ diferiu estatisticamente do tratamento C. flexuosus $30 \%$. O teste B. trimera $20 \%$ apresentou a maior proporção de germinação de sementes duras, diferindo estatisticamente de todos os demais tratamentos. Houve redução significativa das micotoxinas com o uso de todos os OE testados, embora nenhuma tenha sido superior ao tratamento com Vitavax + Thiran® (zero). Os óleos essenciais mostram eficácia na redução de patógenos na semente de soja, e algumas amostras proporcionaram incremento no rendimento de grãos, mostrando a necessidade de maiores estudos acerca do uso destes, na busca de correlacionar o constituinte e o efeito farmacológico, bem como formas de melhor aproveitamento do mesmo no tratamento de grãos como perspectiva de novos alternativas farmacológicas.

PALAVRAS-CHAVE: Cymbopogon flexuosus Stapf, Eucalyptus globulus Labill., Baccharis trimera (Less) DC, Glicine max (L.) Merrill.

\section{EFFECTIVENESS EVALUATION OF ESSENTIAL OILS ON SOYBEAN SEED TREATMENT}

ABSTRACT: The objective was to evaluate the effect of the essential oils (OE) Cymbopogon flexuosus Stapf, Eucalyptus globulus Labill. and Baccharis trimera (Less) DC. on phytopathogenic microflora and soybean physiology, OE application at concentrations of 10,20 and 30\%. Analyses of germination and vigor were carried in the Analysis Laboratory seeds of Regional State University Northwest of Rio Grande do Sul - UNIJUI and treatments deployed to the field in the experimental area of the Regional Institute for Rural Development - IRDeR. The variables: initial and final number of healthy plants, diseased plants, grain yield, thousand grain weight, number of pods and grains per plant, grain weight per plant; biological yield; harvest index. As for in vitro analysis of the seeds was checked: counting normal seedlings germinated; abnormal germinated seedlings count and hard seeds. It used analysis of variance with Tukey test at $5 \%$ probability. Treatment with OE E. globulus $20 \%$ had the highest grain yield, an increase of $10 \%$ compared to control. As for the weight of a thousand grains, the average of all tests was $140 \mathrm{~g}$, showing no statistical difference. As the final number of plants $B$. trimera $30 \%$ differed from treatment $C$. flexuosus $30 \%$. The test $B$. trimera $20 \%$ had the highest proportion of germination of hard seeds, were significantly different from all other treatments. There was a significant reduction of mycotoxins using all tested $\mathrm{OE}$, though none was superior to treatment with Vitavax + Thiran ${ }^{\circledR}$ (zero). Essential oils show effectiveness in reducing pathogens in soybean, and some samples provided increase in grain yield, showing the need for further studies on the use of these, seeking to correlate the constituent and pharmacological effects, and ways to better utilization of the same in the treatment of grains as new pharmacological alternatives.

KEY WORDS: Cymbopogon flexuosus Stapf, Eucalyptus globulus Labill, Baccharis trimera (Less) DC, Glicine max. 


\section{INTRODUÇÃO}

0 aumento da produção de soja e sua consequente expansão nos últimos anos, associada ao monocultivo, favoreceu em grande escala ao aumento na incidência de pragas e doenças. Estas podem acometer a planta durante todas as fases da cultura, reduzindo significativamente a produtividade dessa oleaginosa, limitando a lucratividade e a sustentabilidade da propriedade rural, uma vez que causam redução na germinação e no vigor (Garcia et al., 2012). Dentre os patógenos transmitidos pelas sementes, os fungos são considerados os mais importantes, devido ao maior número, mas também pelos prejuízos causados tanto no rendimento, quanto na qualidade de sementes (Celoto et al., 2008).

0 tratamento realizado diretamente sobre a superfície da semente pode ser uma opção rápida e eficiente no controle destes patógenos, desde que os produtos aplicados não causem impacto ao meio ambiente e sejam efetivos para a proteção contra os microrganismos presentes no solo e a preservação da qualidade da semente durante 0 armazenamento (Ramos et al., 2008). A eficiência do tratamento de sementes, visando o controle de patógenos, depende do tipo e localização do patógeno, do vigor da semente e da disponibilidade de substâncias e processos adequados (Mentem; Moraes, 2010; Queiroga et al., 2012). Este tratamento pode evitar 0 desenvolvimento de epidemias no campo; proporcionar maior sustentabilidade à cultura pela redução de riscos na fase de implantação da lavoura e promover 0 estabelecimento inicial da lavoura com uma população ideal de plantas (França Neto, 2009).

No Brasil, estima-se que $100 \%$ das sementes de soja são tratadas com fungicidas, 30\% com inseticidas e $50 \%$ com micronutrientes com objetivo de proteger o estabelecimento no campo ou até mesmo o seu desenvolvimento vegetativo (Juliatti, 2010). Como alternativa ao uso de tratamentos químicos, têm sido pesquisado produtos naturais, como extratos e óleos essenciais de origem vegetal, que podem apresentar propriedades antimicrobianas capazes de controlar a microflora associada às sementes (Gonçalves et al., 2009).

Poucos trabalhos práticos têm sido realizados para avaliar o efeito dos óleos essenciais no controle de fitopatógenos, e grande parte das pesquisas são conduzidas em uma condição experimental, in vitro, não existindo muitos trabalhos que avaliam a eficácia dos óleos essenciais quando utilizados em sementes cultivadas a campo. Neste contexto, o objetivo do presente estudo foi avaliar a eficácia do tratamento de sementes de soja com óleos essenciais de Cymbopogon flexuosus Stapf (citronela), Eucalyptus globulus Labill. (eucalipto) e Baccharis trimera (Less) DC. (carqueja) em diferentes concentrações em condições experimentais a de campo.

\section{MATERIAL E MÉTODOS}

0 trabalho foi desenvolvido na área experimental do Instituto Regional de Desenvolvimento Rural (IRDeR), da Universidade Regional do Noroeste do Estado do Rio Grande do Sul (UNIJUÍ), em ljuí/RS e os testes para avaliação do efeito dos óleos essenciais nas sementes foram desenvolvidos no Laboratório de Análise de sementes (LAS) da mesma universidade.

Os óleos essenciais utilizados nas formulações foram obtidos das folhas frescas de $C$. flexuosus, $E$. globulus. e B. trimera, extraídos em destilador modelo D20, fabricado pela LINAX. O rendimento obtido na extração dos óleos essenciais foi de $1,6 \%$ para $E$. globulus, $1,2 \%$ para $C$. flexuosus e $0,5 \%$ para $B$. trimera.

As análises qualitativas dos óleos essenciais foram realizadas por cromatografia em fase gasosa (CG) e por cromatografia em fase gasosa acoplada a espectrometria de massa (CG/ EM). Para a cromatografia gasosa utilizou-se um cromatógrafo Variam 3400 acoplado a um injetor automático de amostra Variam 8200 CX, com detector de ionização de chama (DIC).

A cultivar de soja utilizada foi a FPS JÚPITER $\mathrm{RR}$, sendo os estudos realizados na safra agrícola de 2012/2013. O delineamento experimental foi em blocos ao acaso com quatro repetições, envolvendo tratamentos de sementes com óleos essenciais comparados com controle positivo e controle negativo.

Os óleos essenciais foram preparados em soluções nas concentrações de 10, 20 e 30\%, empregando $1 \%$ de propilenoglicol como agente emulsionante. 0 excipiente utilizado foi água destilada totalizando $50 \mathrm{~mL}$ de solução/Kg, sendo os testes: T1 - Controle negativo; T2 - Controle positivo - VitavaxThiram® 200 SC; T3 Cymbopogon flexuoxus (CB) 10\%; T4 - CB 20\%;; T5 - CB 30\%; T6 - Eucalyptus globulus (EG) 10\%; T7 - EG 20\%; T8 - EG 30\%; T9 
- Baccharis trimera (BT) 10\%; T10 - BT 20\% (T10); T11 - BT 30\%.

As parcelas experimentais foram constituídas por 6 linhas de 5 metros de comprimento, com espaçamento de 0,50 metros entre fileiras, sendo considerada como parcela útil às quatro fileiras centrais.

Para 0 tratamento das sementes 0 método empregado foi pulverização manual, até 0 escorrimento do produto sobre as mesmas. Após a pulverização as sementes foram secadas a temperatura ambiente em recipiente aberto, sendo semeadas 24 horas após o tratamento.

0 teste de germinação foi realizado segundo as Regras para Análise de Sementes (RAS) (Brasil, 2009). Foram utilizadas 400 sementes para cada tratamento, sendo oito repetições de 50 sementes, incubadas em rolo de papel Germitest $₫$, umedecidos com água destilada (volume três vezes superior ao peso do papel) e incubadas em germinadores, sob uma temperatura de $25^{\circ} \mathrm{C}$ e luz permanente. A avaliação da germinação foi realizada no sétimo dia de incubação e verificou-se: 1) contagem de plântulas germinadas normais (que apresentaram as estruturas vegetativas perfeitas); 2) contagem de plântulas germinadas anormais (que apresentaram as estruturas vegetativas imperfeitas); 3 ) sementes mortas (que não apresentaram germinação).

No teste de vigor das sementes ou envelhecimento acelerado tradicional (EAT) as amostras foram colocados em câmera de envelhecimento acelerado em um compartimento com água em torno de $100 \%$ de umidade a $41^{\circ} \mathrm{C}$ durante 48 horas, após esse tratamento foi realizado o plantio das sementes, foram utilizadas 8 repetições de 50 sementes alocado, conforme metodologia adaptada de Vieira et al. (1992).

Para 0 teste de patologia foram utilizadas 200 sementes por tratamento, sendo 4 repetições de 50 sementes, utilizando-se caixas Gerbox®, contendo três folhas de papel filtro umedecidas com água destilada. As caixas com as sementes foram colocadas em câmara de germinação na câmara em temperatura de mais ou menos $20^{\circ} \mathrm{C} \pm 2$ com fotoperíodo de 12 horas, por 7 dias. Após este período avaliou-se individualmente cada semente com auxílio de microscópios estereoscópicos e óptico identificando os fungos a ela associados, conforme metodologia de Brasil (2009).

0 experimento conduzido em campo não utilizou irrigação, seguindo a época recomendada para semeadura da cultura de acordo com as indicações técnicas para a cultura da Soja no Rio Grande do Sul e em Santa Catarina, safras 2012/2013 e 2013/2014. A área na qual foi instalado 0 experimento tem o sistema de semeadura direta consolidada, implantado sobre o precedente cultural de aveia branca (Avena sativa L.).

Aárea experimental foi previamente dessecada, sendo este procedimento realizado cerca de vinte dias antes da semeadura, com Nortox 2,4-D® (2,4-D Amina $806 \mathrm{~g} / \mathrm{L}$ ) e Roundup Transorb® (Glifosato $580 \mathrm{~g} / \mathrm{L}$ ), na dose de $1,5 \mathrm{~L} / \mathrm{ha}^{-1}$ e $2,5 \mathrm{~L} / \mathrm{ha}^{-1}$ respectivamente, na vazão de 100 litros de água/ha.

A adubação foi realizada no sulco de semeadura com o uso de uma semeadeira mecânica para plantio direto, na base de $200 \mathrm{~kg} / \mathrm{ha}^{-1}$ de adubo químico da fórmula 2-23-23 $\left(\mathrm{N}-\mathrm{P}_{2} \mathrm{O}_{5}-\mathrm{K}_{2} \mathrm{O}\right)$, para uma expectativa de produtividade de grãos de $3 \mathrm{tha}^{-1}$.

A semeadura foi realizada no dia 21 de novembro de 2012 com auxílio de uma semeadeira manual adaptada, proporcionando uma melhor homogeneidade das sementes por unidade de área, regulada para distribuição de 12 sementes por metro linear, numa profundidade de 4 a $5 \mathrm{~cm}$. No dia 03 de dezembro de 2012 as plantas já tinham emergido e realizou-se a avaliação do número total de plantas iniciais de cada parcela e a incidência de manchas nos cotilédones destas, contando apenas as 4 linhas centrais da parcela.

0 controle de plantas daninhas foi realizado com aplicação de Roundup Transorb® (Glifosato 580 $\mathrm{g} / \mathrm{L})$, em pós-emergência, na dose de $1,5 \mathrm{~L} \mathrm{ha}^{-1}$, e para controle de lagartas foi usado o inseticida Curyom 550 EC@ (Profenofós 500g/L+Lufenuron 50g/L)nadosagem de $400 \mathrm{~mL} / \mathrm{ha}$, este procedimento foi realizado quando a soja estava no estádio V4. No estádio R1 foi realizado uma aplicação com fungicida Priori Xtra $\circledast$ (Azoxistrobina 200g/L + Ciproconazol 80g/L) na dosagem de 300 $\mathrm{mL} / \mathrm{ha}$ juntamente com inseticida Curyom $550 \mathrm{EC} \odot$ (Profenofós 500g/L + Lufenuron $50 \mathrm{~g} / \mathrm{L}$ ) com $400 \mathrm{~mL} /$ ha, a última aplicação de tratamentos foi no estágio R5, no qual foi usado fungicida Priori Xtra $₫$ (Azoxistrobina $200 \mathrm{~g} / \mathrm{L}+$ Ciproconazol $80 \mathrm{~g} / \mathrm{L}) 300 \mathrm{~mL} / \mathrm{ha}$ de produto e inseticida Karate zeon 250 CS 8 (Lambda-cialotrina $250 \mathrm{~g} / \mathrm{L})$ na dosagem de $30 \mathrm{~mL} / \mathrm{ha}$, já o controle de plantas invasoras nos estádios subsequentes a ultima aplicação de herbicida, foram realizadas de acordo com a necessidade, mediante capina manual. 
No dia 07 de abril de 2013 foi realizada a contagem final das plantas em cada parcela, no dia 08 de abril de 2013 foi coletado em cada parcela as três melhores linhas centrais das quatro úteis, para posterior mensuração dos componentes de rendimento.

As variáveis determinadas foram: 1) Número de plantas inicial (NPI); 2) Número de plantas final (NPF); 3) Número de plantas doentes (NPD); 4) Rendimento de grãos (RG); 4) Massa de mil grãos (MMG); 5) Número de legumes por planta (NLP); 5) Número de grãos por planta (NGP); 6) Peso de grãos por planta (PGP); 7) Rendimento biológico aparente (RBA); 8) Índice de colheita (IC).

Os dados foram submetidos à análise de variância pelo programa Genes. Para variáveis descritivas foi utilizada média e desvio padrão, para variáveis associativas com teste de Tukey a $5 \%$ de probabilidade.

\section{RESULTADOS E DISCUSSÃO}

Quanto aos constituintes químicos dos óleos essenciais (OE) utilizados, observou-se que $C$. flexuosus apresentou $81,79 \%$ da sua composição de citral. $O$ óleo essencial de B. Trimera $54,37 \%$ de acetato de carquejita, 7,39\% de a-Pineno e 5,38\% de limoneno e por fim, o E. globulus $16,38 \%$ de a-Pineno, $4,54 \%$ de limoneno e 76,02 de cineol. Os dados estão de acordo com estudos que apontam que OE extraído das folhas de $C$. flexuosus apresenta como principal constituinte 0 citral, e em menores quantidades o limoneno, citronelal, ß-mirceno e geraniol (Silveira et al. 2012). Já para espécies de Baccharis a composição química destes óleos essenciais é muito variada (Souza et al. 2011).

A análise de comparação de médias para os caracteres RG, MMG, NLP, NGP, encontra-se na Tabela 1. No RG podemos observar variação entre os tratamentos, sendo evidenciado que o tratamento E.globulus 20\% (3697 Kg/ha) e B. trimera 10\% (3618 Kg/ha) foram superiores, entretanto, diferiram estatisticamente somente do tratamento $C$. flexuosus $30 \%$ (3092 Kg/ha). Um comparativo do percentual de RG do E. globulus 20\% apresentou um ganho de $10 \%$ com relação ao controle. Os tratamentos E. globulus $30 \%$ e C. flexuosus 30\% tiveram rendimento de grãos menor que o controle, entretanto não houve diferença estatística entre os mesmos. No tratamento $C$. flexuosus 0 aumento da concentração do óleo essencial provocou diminuição no RG (Tabela 1).

Tabela 1. Rendimento de grãos (RG), massa de mil grãos (MMG), número de legume por planta (NLP), número de grãos por planta (NGP) de soja submetida ao tratamento de sementes com óleos essenciais e Vitavax-Thiran®. IRDeR/DEAg.UNIJUÍ. Augusto Pestana. RS.2013.

\begin{tabular}{|c|c|c|c|c|c|}
\hline \multirow{2}{*}{ Tratamento } & \multicolumn{2}{|l|}{ RG } & \multirow{2}{*}{$\begin{array}{c}\text { MMG } \\
\text { G }\end{array}$} & \multirow{2}{*}{$\begin{array}{c}\text { NLP } \\
n^{0}\end{array}$} & \multirow{2}{*}{$\begin{array}{c}\text { NGP } \\
\mathrm{n}^{0}\end{array}$} \\
\hline & $\mathrm{Kg} / \mathrm{ha}^{-1}$ & $\%$ & & & \\
\hline E. globulus 20\% & S $3697 a^{*}$ & 100 & S $145 a^{*}$ & $75 a^{*}$ & $159 a^{*}$ \\
\hline B. trimera $10 \%$ & $3618 \mathrm{a}$ & 98 & $142 a$ & $\mathrm{~S} 100 \mathrm{a}$ & S $210 a$ \\
\hline B.trimera $30 \%$ & $3566 \mathrm{ab}$ & 96 & $140 a$ & $172 a$ & I $156 \mathrm{a}$ \\
\hline E. globulus $10 \%$ & $3530 \mathrm{ab}$ & 95 & $142 a$ & $81 \mathrm{a}$ & $182 \mathrm{a}$ \\
\hline B. trimera $20 \%$ & $3529 a b$ & 95 & $140 a$ & $83 a$ & $179 a$ \\
\hline C. flexuosus 10\% & $3520 \mathrm{ab}$ & 95 & I $137 \mathrm{a}$ & $76 a$ & $168 \mathrm{a}$ \\
\hline C. flexuosus 20\% & $3501 \mathrm{ab}$ & 95 & I 134 a & $82 a$ & $175 a$ \\
\hline Vitavax + Thiran® & $3436 \mathrm{ab}$ & 93 & S143a & $87 a$ & $186 \mathrm{a}$ \\
\hline Controle & $3350 \mathrm{ab}$ & 90 & $142 \mathrm{a}$ & $78 a$ & $171 \mathrm{a}$ \\
\hline E. globulus 30\% & $3339 a b$ & 90 & $140 a$ & $87 a$ & $188 \mathrm{a}$ \\
\hline C. flexuosus 30\% & I 3092 b & 84 & $140 a$ & $\mathbf{S} 90 \mathrm{a}$ & S193a \\
\hline Média & 3471 & - & 140 & 82 & 178 \\
\hline CV\% & 6 & - & 5 & 20 & 22 \\
\hline Desvio padrão & 163 & - & 3 & 8 & 15 \\
\hline
\end{tabular}

* Médias seguidas pelas mesmas letras na coluna não diferem entre si pelo Teste de Tukey a $5 \%$ de probabilidade. S - Superior à média mais desvio padrão. I - Inferior à média menos desvio padrão. 
Analisando a MMG podemos constatar que a média de todos os tratamentos foi $140 \mathrm{~g}$ e não evidenciou diferenças entre os mesmos, a mesma coisa foi observada para as variáveis NLP e NGP (Tabela 1).

Para o NPI nota-se variação entre tratamentos, apresentando um maior percentual de plantas o tratamento B. trimera $30 \%$ e $20 \%$, bem como o tratamento Vitavax-Thiran $\circledast$, porém estes não diferiram estatisticamente do controle. 0 menor NPI foi observado no tratamento C. flexuosus $30 \%$, sendo estatisticamente inferior ao tratamento com Vitavax + Thiran® (Tabela 2).

Tabela 2. Número de plantas iniciais (NPI), número de plantas final (NPF), rendimento biológico aparente (RBA), peso de grãos por planta (PGP) e índice de colheita (IC) de soja submetida ao tratamento de sementes com óleos essenciais e Vitavax-Thiran®. IRDeR/DEAg/UNIJUÍ. Augusto Pestana. RS. 2013.

\begin{tabular}{|c|c|c|c|c|c|c|c|}
\hline \multirow{2}{*}{ Tratamento } & \multirow{2}{*}{$\begin{array}{c}\text { NPI } \\
\mathrm{n}^{0}\end{array}$} & \multicolumn{3}{|c|}{ NPF } & \multirow{2}{*}{$\begin{array}{c}\text { RBA } \\
\mathrm{g}\end{array}$} & \multirow{2}{*}{$\begin{array}{c}\text { PGP } \\
\mathrm{g}\end{array}$} & \multirow{2}{*}{$\begin{array}{l}\text { IC } \\
\% \\
\end{array}$} \\
\hline & & $\%$ & $\mathrm{n}^{0}$ & $\%$ & & & \\
\hline E. globulus 20\% & $37 a b$ & 90 & $34 a b$ & 89 & $43 a^{*}$ & $23 a^{*}$ & S54a* \\
\hline B. trimera 10\% & $37 a b$ & 90 & $33 a b$ & 87 & S55a & S29a & S54a \\
\hline B. trimera 30\% & S41a & 100 & S38a & 100 & $142 a$ & $122 \mathrm{a}$ & $53 a$ \\
\hline E. globulus $10 \%$ & I33ab & 80 & $30 a b$ & 79 & $48 a$ & S26a & S55a \\
\hline B. trimera $20 \%$ & $\mathrm{~S} 40 \mathrm{a}$ & 98 & $34 a b$ & 89 & $47 a$ & $25 a$ & $53 a$ \\
\hline C.flexuosus $10 \%$ & S39ab & 95 & s35ab & 92 & $43 a$ & $23 a$ & $53 a$ \\
\hline C.flexuosus $20 \%$ & $37 a b$ & 90 & $33 a b$ & 87 & $45 a$ & $23 a$ & $152 a$ \\
\hline Vitavax+Thiran® & S41a & 100 & $33 a b$ & 87 & $49 a$ & $\mathbf{S} 27 \mathrm{a}$ & S54a \\
\hline Controle & $35 a b$ & 85 & $31 a b$ & 82 & $44 a$ & $24 a$ & S55a \\
\hline E.globulus 30\% & $35 a b$ & 85 & $30 a b$ & 79 & $49 a$ & $26 a$ & S54a \\
\hline C. flexuosus $30 \%$ & $129 b$ & 71 & $125 b$ & 66 & S52a & $\mathbf{S} 27 \mathrm{a}$ & $152 \mathrm{a}$ \\
\hline Média & 36 & - & 32 & - & 46 & 24 & 53 \\
\hline $\mathrm{CV} \%$ & 11 & - & 12 & - & 20 & 20 & 3 \\
\hline Desvio padrão & 3 & - & 3 & - & 4 & 2 & 1 \\
\hline
\end{tabular}

* Médias seguidas pelas mesmas letras na coluna não diferem entre si pelo Teste de Tukey a $5 \%$ de probabilidade. S - Superior à média mais desvio padrão. I - Inferior à média menos desvio padrão.

Para NPF o maiorpercentual foi para o tratamento B. trimera $30 \%$ (38) que diferiu estatisticamente do tratamento C. flexuosus 30\%, apresentando menor NPF. Em um estudo avaliando o efeito repelente e inseticida do óleo essencial de B. trimera sobre Coleópteros, Scariot et al. (2013) observaram desempenho positivo do óleo essencial de $B$. trimera como atividade inseticida e repelente. Provavelmente o maior NPI e NPF observado neste estudo, foi resultado da redução de pragas de solo pelo óleo essencial.

O potencial herbicida dos óleos voláteis, inibindo a germinação de sementes e 0 crescimento de plântulas, pode ser atribuído a um dos constituintes químicos do óleo ou a interação dos seus componentes (An et al. 1993).

Observa-se que a análise comparativa de médias não detectou diferenças significativas para as variáveis RBA, PGP e IC (Tabela 2).

Nota-se que os tratamentos B. trimera $20 \%$ e E. globulus $30 \%$ foram os que apresentaram menor GERM (Tabela 3), esses tratamentos também apresentaram o maior número de micotoxinas (Tabela 4). Isto porque, a presença de micotoxinas afeta a qualidade das sementes por reduzir o seu poder germinativo (Machado, 2000).

Analisando a Tabela 3, pode-se observar diferença estatística entre os tratamentos nas variáveis GERM, GERAN, GERMOR, VIGOR, VIGAN e VIGDUR. $O$ tratamento $B$. trimera $30 \%$ exibiu maior percentual de plantas GERM, porém este valor não diferiu estatisticamente do controle negativo e do tratamento com Vitavax + Thiran®. 0 tratamento $B$. trimera 20\% apresentou menor GERM (63\%), sendo estatisticamente inferior ao controle e ao Vitavax + Thiran®. $O$ efeito alelopático de extratos de carqueja já foi constatado em sementes de outras plantas, como é o caso do picão-preto (Bidens pilosa L.), neste estudo verificou-se a redução da porcentagem e da velocidade de germinação dessas sementes (Depiné, 2003). 
Tabela 3. Germinação de sementes \% (GERM), Germinação plantas anormais \% (GERAN), germinação sementes mortas \% (GERMOR), vigor \% (VIGOR), vigor das sementes anormais (VIGAN) e vigor sementes duras (VIGDUR) submetidas ao tratamento de sementes com óleos essenciais e Vitavax-Thiran®. IRDeR/DEAg/UNIJUÍ. Augusto Pestana. RS.2013.

\begin{tabular}{ccccccc}
\hline Tratamento & GERM & GERAN & GERMOR & VIGOR & VIGAN & \multicolumn{2}{c}{ VIGDUR } \\
& $\%$ & $\%$ & $\%$ & $\%$ & $\%$ & $\%$ \\
\hline E.globulus 20\% & $69 \mathrm{abc} c^{*}$ & $30 \mathrm{a}^{*}$ & $1 \mathrm{~b}^{*}$ & $61 \mathrm{ab}$ & $32 \mathrm{a}^{*}$ & $7 \mathrm{~b}^{*}$ \\
B.trimera 10\% & $70 \mathrm{abc}$ & $28 \mathrm{ab}$ & $2 \mathrm{~b}$ & $61 \mathrm{ab}$ & $30 \mathrm{ab}$ & $9 \mathrm{ab}$ \\
B.trimera 30\% & $77 \mathrm{a}$ & $21 \mathrm{~b}$ & $2 \mathrm{~b}$ & $63 \mathrm{ab}$ & $29 \mathrm{ab}$ & $8 \mathrm{ab}$ \\
E.globulus 10\% & $71 \mathrm{ab}$ & $27 \mathrm{ab}$ & $2 \mathrm{~b}$ & $58 \mathrm{~b}$ & $30 \mathrm{ab}$ & $12 \mathrm{ab}$ \\
B.trimera 20\% & $63 \mathrm{c}$ & $25 \mathrm{ab}$ & $12 \mathrm{a}$ & $61 \mathrm{ab}$ & $23 \mathrm{~b}$ & $16 \mathrm{a}$ \\
C.flexuosus 10\% & $72 \mathrm{ab}$ & $27 \mathrm{ab}$ & $1 \mathrm{~b}$ & $67 \mathrm{ab}$ & $27 \mathrm{ab}$ & $6 \mathrm{~b}$ \\
C.flexuosus 20\% & $71 \mathrm{ab}$ & $27 \mathrm{ab}$ & $2 \mathrm{~b}$ & $68 \mathrm{a}$ & $28 \mathrm{ab}$ & $6 \mathrm{~b}$ \\
Vitavax + Thiran® & $74 \mathrm{ab}$ & $24 \mathrm{ab}$ & $2 \mathrm{~b}$ & $63 \mathrm{ab}$ & $28 \mathrm{ab}$ & $9 \mathrm{ab}$ \\
Testemunha & $75 \mathrm{ab}$ & $24 \mathrm{ab}$ & $1 \mathrm{~b}$ & $63 \mathrm{ab}$ & $29 \mathrm{ab}$ & $8 \mathrm{ab}$ \\
E.globulus 30\% & $68 \mathrm{bc}$ & $29 \mathrm{ab}$ & $3 \mathrm{~b}$ & $61 \mathrm{ab}$ & $31 \mathrm{ab}$ & $8 \mathrm{ab}$ \\
C.flexuosus 30\% & $69 \mathrm{abc}$ & $27 \mathrm{ab}$ & $4 \mathrm{~b}$ & $66 \mathrm{ab}$ & $24 \mathrm{ab}$ & $10 \mathrm{ab}$ \\
\hline Média & 71 & 26 & 3 & 63 & 28 & 9 \\
CV\% & 2 & 6 & 24 & 3 & 7 & 17 \\
\hline
\end{tabular}

* Médias seguidas pelas mesmas letras na coluna não diferem entre si pelo Teste de Tukey a 5\% de probabilidade.

Otratamentoqueapresentou omenorpercentual de GERAN foi o B. trimera 30\% (21\%), diferindo estatisticamente apenas do teste E. globulus 20\%. 0 tratamento C. flexuosus 10, 20 e 30\% apresentaram as mesmas porcentagens de GERAN (27\%), não diferindo estatisticamente do controle (Tabela 3). 0 teste $B$. trimera $20 \%$ apresentou a maior proporção de GERMOR (12\%), diferindo estatisticamente de todos os demais tratamentos (Tabela 3).

Com relação ao vigor o maior valor obtido foi o C. flexuosus $20 \%$, contudo diferindo estatisticamente apenas do E. globulus 10\% (58\%). O maior VIGAN foi obtido com E. globulus 20\% (32\%) diferindo estaticamente de B. trimera $20 \%$ (23\%), desta forma este apresentou maior percentagem de plantas anormais no teste de vigor. Com relação ao VIGDUR o tratamento $B$. trimera $20 \%$ registrou maior número de sementes duras $(16 \%)$, enquanto para o $E$. globulus $20 \%$ e $C$. flexuosus $10 \%$ e $20 \%$ verificou-se os menores valores, não diferindo do Vitavax + Thiran ${ }^{\circledR}$.

Os efeitos dos aleloquímicos nos diferentes processos fisiológicos de uma planta são dependentes das concentrações e o efeito sobre as sementes poderia ocorrer por meio de atividade específica sobre sementes menos vigorosas ou mesmo, indistintamente, em toda população (Reigosa et al. 1999). Ainda Teixeira (2005) afirmou que a ação das substâncias aleloquímicas não é muito específica, podendo uma mesma substância desempenhar várias funções, dependendo de sua concentração e forma de translocação na planta.

$\mathrm{Na}$ Tabela 4, observamos que os óleos testados inibiram o crescimento de Fusarium sp., comparado com 0 controle, porém, apenas 0 tratamento com Vitavax + Thiran ${ }^{\circledR}$ erradicou completamente 0 patógeno. Uma análise do percentual de redução de Fusarium sp. indica que os óleos essenciais reduziram em até $52 \%$ a incidência do fungo, quando comparado com o controle.

Houve redução significativa das micotoxinas com o uso de todos os OE testados, sendo que a maior redução foi obtida com os tratamentos Vitavax + Thiran® (zero) e C. flexuosus 10\% (4), não diferindo estatisticamente entre si, mas diferindo do controle (Tabela 4). 
Tabela 4. Fusarium sp., Micotoxinas e número de plantas doentes (NPD) de Soja submetidas ao tratamento de sementes com óleos essenciais e Vitavax + Thiran®. IDeR/DEAg/UNIJUÍ. Augusto Pestana. RS. 2013.

\begin{tabular}{|c|c|c|c|c|c|}
\hline \multirow{2}{*}{ Tratamento } & \multicolumn{2}{|c|}{ Fusarium sp. } & \multicolumn{2}{|c|}{ Micotoxinas $^{1}$} & \multirow{2}{*}{$\frac{\text { NPD }}{n^{0}}$} \\
\hline & $n^{0}$ & $\%$ & $n^{0}$ & $\%$ & \\
\hline E.globulus 20\% & $11 a^{*}$ & 48 & $14 \mathrm{bc}^{*}$ & 45 & $3 a^{*}$ \\
\hline B.trimera 10\% & $12 \mathrm{a}$ & 52 & $18 a b$ & 58 & $4 a$ \\
\hline B.trimera $30 \%$ & $15 a$ & 65 & $17 a b$ & 55 & $4 a$ \\
\hline E.globulus $10 \%$ & $15 a$ & 65 & $13 b c$ & 42 & $4 a$ \\
\hline B. trimera 20\% & $14 a$ & 61 & $31 a$ & 100 & $3 a$ \\
\hline C. flexuosus $10 \%$ & $20 a$ & 87 & $4 c d$ & 13 & 3a \\
\hline C. flexuosus 20\% & $11 a$ & 48 & $13 b c$ & 42 & $3 a$ \\
\hline Vitavax + Thiran $®$ & $0 b$ & 0 & Od & 0 & $3 a$ \\
\hline Controle & $23 a$ & 100 & $9 b c$ & 29 & 3a \\
\hline E. globulus 30\% & $11 a$ & 48 & $22 a b$ & 71 & $3 a$ \\
\hline C. flexuosus 30\% & $13 a$ & 57 & $18 a b$ & 58 & $3 a$ \\
\hline Média & 13 & - & 14 & - & 3 \\
\hline $\mathrm{CV} \%$ & 21 & - & 17 & - & 19 \\
\hline
\end{tabular}

${ }^{1}$ Aspergillus sp. e Penisillium sp.

* Médias seguidas pelas mesmas letras na coluna não diferem entre si pelo Teste de Tukey a $5 \%$ de probabilidade.

Alguns autores como Mieth et al. (2007) e Souza et al. (2010) comprovam a eficiência de produtos naturais no controle de patógenos e no aumento do poder germinativo de sementes.

Em trabalho desenvolvido na Embrapa Clima Temperado em Pelotas-RS foram avaliados diferentes óleos essenciais para controle de Phytophthora infestans em três cultivares de batata inglesa. Possibilitando observar que óleo de camomila inibiu a severidade de doença nas cultivares BINTJE e ELIZA, o óleo de orégano inibiu a severidade de doenças na cultivar ELIZA, entretanto esses óleos não alteraram a produção independentemente da cultivar testada (Viridiana et al. 2006). 0 trabalho avaliou também os óleos essenciais de $E$. globulus e $C$. citratus, entretanto tiveram um comportamento inferior na inibição do crescimento micelial do patógeno quando comparado com o óleo essencial de camomila.

Em pesquisa realizada na Índia foi avaliada a atividade fungitóxica do Citral (constituinte químico majoritário no C. flexuosus) sobre Penicillium sp., Alternaria sp., Fusarium sp., Aspergillus e Butritis nas concentrações de 500 ppm e 1500 ppm, sendo constatado total inibição de crescimento fúngico na maior concentração testada (Bettiol; Morandi, 2009).

Em estudo avaliando a atividade fungitóxica do óleo essencial de Eucaliptus citriodora, Eucalyptus camaldulensis e Eucalyptus urophylla sobre Fussarium sp., Salgado et al. (2003) observaram redução micelial por todos os produtos testados. Esta atividade foi atribuída ao sesquiterpeno globulol.

Em tratamento de sementes de soja utilizando óleos essenciais de Ocimum sp., C. flexuosus, C. citratus e Melaleuca sp. Morais et al. (2008) observaram com relação a sanidade, que não houve diferenças estatísticas significativas entre os tratamentos testados no controle de Fusarium spp., Penicillium spp. e Alternaria sp. indicando que esses óleos não tiveram efeito sobre controle desses patógenos no experimento realizado. Os óleos essenciais de C. citratus, $C$. flexuosus e Melaleuca sp. reduziram a ocorrência de Phomopsis sp. nas sementes, e o óleo essencial de $C$. flexuosus apresentou atividade inibitória sobre Colletotrichum sp. Mostrando que os resultados do presente estudo foram mais positivos e promissores.

A melhor resposta em RG obtidas pelos tratamentos E. globulus $20 \%$ e B. trimera $10 \%$, que poderia ser atribuída a redução da incidência de Fusarium sp. nas sementes, que resultaria em um efeito fisiológico benéfico no desenvolvimento das plantas e na sua produção. Isto pode ser entendido porque os óleos essenciais apresentam uma diversidade de constituintes químicos na sua composição, e muitas vezes não existem estudos que comprovem a efetividade dos constituintes, nem a interação entre eles. 
Sementes predispostas à ação de microrganismos, quando tratadas, reduzem a capacidade de sobrevivência dos fitopatógenos e potencializam a longevidade das sementes, seu poder germinativo e o vigor das futuras plantas (Carvalho et al. 1999). O que pode ser observada em alguns produtos testados.

Outra metodologia aplicada foi a análise para separação de grupos de tratamentos, sendo o que apresentou rendimento de grãos superior ao valor da média acrescido de um desvio padrão, e inferior quando o tratamento apresentou valor inferior a média geral menos um desvio padrão. A mesma metodologia foi utilizada para os demais caracteres de importância agronômica. A superioridade no RG do tratamento constituído pela aplicação de E. globulus 20\% (3697 Kg/ha) poderia ser atribuída a MMG e IC que apresentaram superioridade para o caráter (Tabela 02). A mesma reflexão pode ser aplicada para explicar o RG obtido pelo tratamento $B$. trimera $10 \%$ que revelou NLP, NGP, RBA, PGP e IC também superiores. O IC que se constitui na fração dos grãos produzidos em relação à matéria seca total da planta, pode ser empregado em programas de melhoramento genético e na avaliação de genótipos submetidos a diferentes condições de ambiente. O IC indica a habilidade de um genótipo em combinar elevada capacidade de produção e de destinar a matéria seca acumulada aos componentes do rendimento de interesse econômico. Este índice apresenta forte interação Genótipo x Ambiente (Donald; Hamblin, 1976).

Apesar da pequena diferença entre os tratamentos podemos observar que os resultados encontrados com a utilização de óleos essenciais no tratamento de sementes foram semelhantes ao controle positivo (Vitavax + Thiran®).

A utilização de óleos essenciais de espécie aromáticas e medicinais, isolados ou em combinação com outros métodos, poderá ter um importante papel no controle de fitopatógenos, contribuindo para a redução do uso de fungicidas e consequentemente, um menor impacto ao ambiente (Mota e Pessoa, 2003). Esses produtos podem ter ação fungitóxica na inibição da germinação de esporos e crescimento micelial ou até indução de produção de fitoalexinas ou outros compostos de defesa da planta. Entretanto, deve-se considerar que estes princípios ativos, mesmos sendo naturais, são substâncias químicas e devem ser usadas com critério, especialmente em sementes.

Os resultados deste estudo mostram perspectivas de uso de óleos essenciais no tratamento de sementes de soja, considerando que os resultados de eficácia contra patógenos foi semelhante ao controle positivo, e alguns produtos apresentaram rendimento superior na produção de grãos.

Os óleos essenciais reduziram a incidência de Fusarium sp. nas sementes de soja, bem como de Micotoxinas, com o uso de todos os OE testados. Além disso, os resultados demonstram que os óleos essenciais mostram eficácia na redução de patógenos na semente de soja, e algumas amostras proporcionaram incremento no rendimento de grãos. Mostrando a necessidade de maiores estudos acerca do uso destes OEs, na busca de correlacionar o constituinte e o efeito farmacológico, bem como formas de melhor aproveitamento do mesmo no tratamento de grãos, como perspectiva de novas alternativas farmacológicas.

$\mathrm{O}$ uso e pesquisas com OE visa desenvolver produtos que possam ser eficazes, seguros para 0 tratamento de sementes, que possam reduzir o impacto ambiental decorrentes do uso de fungicidas.

\section{REFERÊNCIAS BIBLIOGRÁFICAS}

An, M., Johnson, I.R.; Lovett, J.V. Mathematical modeling of allelopathy: biological response to allelochemicals and its interpretation. Journal of Chemical Ecology, $1993,19,10,2379-88$.

Scariot, M.A., Reichert, F.W.J.; De Campos, A.C.T; Ecker, S.L.; Mossi, A.J.; Radünz, L.L. Efeito repelente e inseticida do óleo essencial de Baccharis trimera (Less.) DC no controle de Acanthoscelides obtectus (Say) (Coleoptera: bruchidae), ANAIS do II SEPE Seminário de Ensino, Pesquisa e Extensão da UFFS Universidade Federal da Fronteira Sul. 2013.

Bettiol, W; Morandi, M.A.B. Biocontrole de doenças de plantas: uso e perspectivas. Jaguariúna: Embrapa Maio Ambiente. 2009. 341p.

Bonna, T.D.M.M. Óleo essencial de orégano, alecrim, canela e extrato de pimento no controle de Salmonella, Eimeria e Clostridium em frangos de corte. Pesquisa Veterinária Brasileira. 2012, 32, 5. 411-418. 
Brasil. Ministério da Agricultura e Reforma Agrária. Regras para análise de sementes. Brasília: SNDA/ DNDV/CLAV, 1992. 365p.

Campanhola, C.; Bettiol, W. Métodos alternativos de controle de fitossanitário. Jaguariúna: EMBRAPA, 2003. $279 p$

Carvalho, R. A.; Choairy, S. A.; Lacerda, J. T.; Oliveira, E. F. Effect of plants with antibiotic properties on the control of Fusarium sp. In.: Anais International Plant Protection Congress, vol 2, Jerusalém. Anais... Israel: Jerusalém, 1999.

Celoto, M. I. B.; Papa, M. F. S.; Sacramento, L. V. S.; Celoto, F. J. Atividade antifúngica de extratos de plantas a Colletotrichum gloeosporioides. Acta Scientiarum Agronomy, 2008, 30, 1, 1- 5.

Depiné, C. Efeito alelopático de carqueja sobre a germinação de plantas daninhas. 43p. Monografia (Bacharelado em Ciências Biológicas) - Núcleo de Ciências Biológicas e da Saúde, Centro Universitário Positivo, Curitiba. 2003.

Donald, C.M.; Hamblin, J. The Biological Yield and Harvest Index of Cereal as Agronomic and Plant Breeding Criteria. Advances in Agronomy, 1976, 28, 361-405.

França Neto, J.B. Evolução do Conceito de Qualidade de Sementes. Informativo ABRATES, 2009, 19, 2, 76-80.

Garcia, R.A., Juliatti, F.C., Barbosa, K.A.G., Cassemiro, T.A. Atividade antifúngica de óleo e extratos vegetais sobre Sclerotinia sclerotiorum. Bioscience Journal, 2012, 28, 1, 48-57.

Gonçalves, G.G.; Mattos, L.P.V.; Morais, L.A.S. Óleos essenciais e extratos vegetais no controle de fitopatógenos de grãos de soja. Horticultura Brasileira, 2009, 27, 2, 102-107.

Hillen, T.; Schwan-Estrada, K.R.F.; Mesquini, R.M.; Cruz, M.E.S.; Stangarlin, J.R.; Nozaki, M. Atividade antimicrobiana de óleos essenciais no controle de alguns fitopatógenos fúngicos in vitro e no tratamento de sementes. Revista Brasileira de Plantas Medicinais, 2012, 14, 3, 439-445.
Inácio, M. M.; Pascualli, L.C.; Zela, S.P.; De Paula, P.R. Diagnóstico de óleos essenciais, sobre 0 desenvolvimento de Phomopsis phaseoli var. sojae, Fusarium sp. e Macrophomina phaseolina. In: Anais $2^{\mathrm{a}}$ Jornada Científica da Unemat. Barra do Burgues, MT. 5 p. 2009.

Jardinetti, V.A.; Cruz, M.E.; Maia, A, J.; Oliveira, S.B.; Santos, E.M. Efeito de óleos essenciais no controle de patógenos de na germinação de sementes de milho (Zea maiys). Anais VII EPCC. Encontro Internacional de Produção cientifica. 25. Maringa-Pr. 2011.

Juliatti, F.C. Avanços No Tratamento Químico De Sementes. Informatico ABRATES. 2010. vol.1, pg 20 23.

Machado, J.C. Patologia de sementes: significado e atribuições. In: Carvalho, NM; Nakagawa J. Sementes: ciência, tecnologia e produção. Jaboticabal: FUNEP, 588p. 2000.

Machado, J.C. Tratamento de Sementes no controle de doenças. Lavras: LAPS/UFLA/FAEPE, 138p, 2000.

Menten, J.O.; Moraes, M.H.D. Tratamento de Sementes: Histórico, Tipos, Características e Benefício. Informatico Abrates. 3, 20, 16-17.2010.

Mieth, A.; Piveta, G.; Pacheco, C.; Hamann, F. A.; Rodrigues, J.; Muniz, M. F. B.; Blume, E. Microflora e qualidade fisiológica de sementes de cedro (Cedrella fissilis) tratadas com extrato natural de hortelã (Mentha piperita). Revista Brasileira de Agroecologia, pg 29-33, 2007.

Morais LAS; Ramos N; Bettiol W; Chaves FCM. Efeito de óleos essenciais na germinação e sanidade de sementes de soja. In: CONGRESSO BRASILEIRO DE OLERICULTURA, Horticultura Brasileira, 2008, 1, 1721.

Mota, J.C.O.; Pessoa, M.N.G. Utilização de óleo essencial e extrato foliar de Lippia sidoides cham. no controle de fungos de sementes de graviola. In: Resumos Expandidos, 36. Anais Congresso Brasileiro de Fitopatologia, Manejo Integrado de Doenças de Plantas, Uberlândia, MG. 2003. 
Neto, A.C.A; Araújo, P.C; Souza, W.C.O; Medeiros, J.G.F; Aguiar, A.V.M. Óleo essencial de anis na incidência e controle de patógenos em sementes de erva-doce (Foeniculum vulgare Mill.). Revista Verde, 2012, 7, 1, 170 - 176.

Queiroga, M. F. C.; Gomes, J. P.; Almeida, F. D. A.; Pessoa, E. B.; Alves, N. Aplicação de óleo no controle de Zabrotes subfasciatus e na germinação de Phaseolus vulgaris. Revista Brasileira de Engenharia Agrícola e Ambiental, 2012, 16, 7, 777-782.

Ramos, N.P.; Marcos Filho J.; Galli, J.A. Tratamento fungicida em sementes de milho doce. Revista Brasileira de Sementes, 2008, 30, 57-61.

Reigosa, M. J., Sanchez-Moreiras A., Gonzales, L. Ecophysiological approach in allelopathy. Critical Reviews in Plant Science, 1999, 18, 5, 577-608.

Salgado, A.P.S.P.; Cardoso, M.G.; Souza, P.E.; Souza, J.A.; Abreu, C.M.P.; Pinto, J.E.B.P. Avaliação da atividade fungitóxica de óleos essenciais de folhas de Eucalyptus sobre Fusarium oxysporum, Botrytis cinerea e Bipolaris sorokiniana. Ciência e Agroecologia, 2003, 27, 249-254.

Silveira, S. M.; cunha júnior, A., Scheuermann, G. N., Secchi, F. L.; Vieira, C. R. W. Chemical composition and antimicrobial activity of essential oils from selected herbs cultivated in the South of Brazil against food spoilage and foodborne pathogens. Ciência Rural, 2012, 42-47.
Souza, P. F.; Silva, G. H.; Henriques, I. G. N., Campelo, G. J; Alves, G. S. Atividade antifúngica de diferentes concentrações de extrato de alho em sementes de ingá (Inga edulis). Revista Verde, 2010, 5, 5, 8-13.

Souza, S.P.; Cardoso, M.G.; Souza, P.E.; Guimarães, L.G.L.; Andrade, J.; Mallet, A.C.T.; Nelson, D.L. Óleo essencial de Baccharis tridentata Vahl: composição química, atividade antioxidante e fungitóxica, e caracterização morfológica das estruturas secretoras por microscopia eletrônica de varredura. Revista Brasileira de Plantas Medicinais, 2011, 13, 4, 456-466.

Teixeira, M. G. Efeito alelopático de extratos alcoólicos cravo-da-índia, canela e noz moscada sobre a germinação de algumas sementes de interesse agronômico. São Paulo: Centro Universitário da Fundação de Ensino Octávio Bastos (UNIFEOB), 67p. Monografia. 2005.

Vieira, R. D.; Carvalho, N. M.; Sader, R. Teste de vigor e suas possibilidades de uso. In: Vieira, R.D.; Carvalho, N. M. (Eds.). Testes de vigor em sementes. Jaboticabal: FCAV/ UNESP, 1992.

Viridiana, K. B., Gomes, C. B., Gomes, J.C.C. Efeito de óleos essenciais de plantas medicinais no controle de Phytophthora infestaus em batata. Revista Brasileira de Agroecologia, 2006, I, 2, 24-32. 